\title{
Paralogous synthetic lethality underlies genetic dependencies of the cancer-mutated gene STAG2
}

\author{
Melanie L Bailey ${ }^{1}$, David Tieu ${ }^{2}$, Andrea Habsid², Amy Hin Yan Tong ${ }^{2}$, Katherine Chan², Jason Moffat ${ }^{2,3,4}$, Philip Hieter ${ }^{1}$ (D
}

\begin{abstract}
STAG2, a component of the mitotically essential cohesin complex, is highly mutated in several different tumour types, including glioblastoma and bladder cancer. Whereas cohesin has roles in many cancer-related pathways, such as chromosome instability, DNA repair and gene expression, the complex nature of cohesin function has made it difficult to determine how STAG2 loss might either promote tumorigenesis or be leveraged therapeutically across divergent cancer types. Here, we have performed whole-genome CRISPR-Cas9 screens for STAG2-dependent genetic interactions in three distinct cellular backgrounds. Surprisingly, STAG1, the paralog of STAG2, was the only negative genetic interaction that was shared across all three backgrounds. We also uncovered a paralogous synthetic lethal mechanism behind a genetic interaction between STAG2 and the iron regulatory gene IREB2. Finally, investigation of an unusually strong context-dependent genetic interaction in HAP1 cells revealed factors that could be important for alleviating cohesin loading stress. Together, our results reveal new facets of STAG2 and cohesin function across a variety of genetic contexts.
\end{abstract}

DOI 10.26508/lsa.202101083 | Received 1 April 2021 | Revised 14 August 2021 | Accepted 16 August 2021 | Published online 30 August 2021

\section{Introduction}

Cohesin is a well-conserved structural ring complex that physically tethers two DNA segments to ensure proper chromatin organization and function. In more complex organisms like humans, cohesin is involved in many diverse cellular functions including sister chromatid cohesion, DNA repair, replication fork restart, transcription, and promotion of topologically associating domains (Losada, 2014; Waldman, 2020). As a multisubunit complex, mitotic cohesin consists of three core ring components, SMC1A, SMC3, and RAD21, as well as several accessory factors including one of two SCC3 paralogs, either STAG1 or STAG2 (Uhlmann, 2016). Although these two paralogs are thought to be mainly interchangeable in the complex, more recent data have indicated separate roles for cohesin-STAG1 and cohesinSTAG2 in a small number of functions (Kong et al, 2014; Daniloski \& Smith, 2017; Kojic et al, 2018; Casa et al, 2020).
In cancer, the STAG2 gene is more highly mutated than any other cohesin component, including STAG1 (Hill et al, 2016; Waldman, 2020). In fact, STAG2 is 1 of only 12 genes significantly recurrently mutated in four or more tumour types (Lawrence et al, 2014) although how STAG2 loss-of-function might promote tumourigenesis remains unclear. Although earlier studies could find no clear correlation between STAG2 mutation and genome instability (BalbásMartínez et al, 2013; Hill et al, 2016; Benedict et al, 2020), loss of STAG2 has more recently been linked to an increase in telomere recombination and a decrease in cell type-specific transcription, both of which could have implications in cellular transformation (Mullenders et al, 2015; Galeev et al, 2016; Daniloski \& Smith, 2017; Kojic et al, 2018). Loss of STAG2 has also been shown to be synthetic lethal with its paralog STAG1 (Benedetti et al, 2017; van der Lelij et al, 2017, 2020; Liu et al, 2018; Viny et al, 2019).

The identification of genetic interactions for a gene of interest, such as a cancer gene, can provide important functional and therapeutic information (O'Neil et al, 2017; Mair et al, 2019). Fortunately, the discovery of CRISPR-Cas9 has made it technically feasible to screen for and test candidate genetic interactions in mammalian systems (Hart et al, 2015; Wong et al, 2016; Behan et al, 2019). In recent years, several groups have proposed a need to incorporate a deeper understanding of the contextdependency of a candidate genetic interaction, specifically the cell type(s) or cell line background(s) where the genetic interaction can be found (Ryan et al, 2018; Shen \& Ideker, 2018). A genetic interaction target observed in many cell backgrounds (i.e., a more context-independent target) has the advantage of not only being a potential therapeutic target across multiple cancer types, but also has a higher likelihood of being more robust in the context of tumour heterogeneity. So far, mechanisms behind context-dependent genetic interactions remain understudied, but recent studies have noted that interactions involving paralogous synthetic lethality are often found in more than one cellular background (Tsherniak et al, 2017; Dede et al, 2020; Gonatopoulos-Pournatzis et al, 2020; Lord et al, 2020). Paralogous synthetic lethality is a genetic interaction that occurs when the loss of one paralog in an essential complex or pathway is buffered by the presence of a second paralog (Muller et al, 2012; D’Antonio et al, 2013; Tsherniak et al, 2017).

Given the importance of STAG2 in cohesin function and its high rate of mutation in diverse types of cancer (Lawrence et al, 2014;

${ }^{1}$ Michael Smith Laboratories, University of British Columbia, Vancouver, Canada ${ }^{2}$ Donnelly Centre, University of Toronto, Toronto, Canada ${ }^{3}$ Department of Molecular Genetics, University of Toronto, Toronto, Canada ${ }^{4}$ Institute of Biomedical Engineering, University of Toronto, Toronto, Canada 


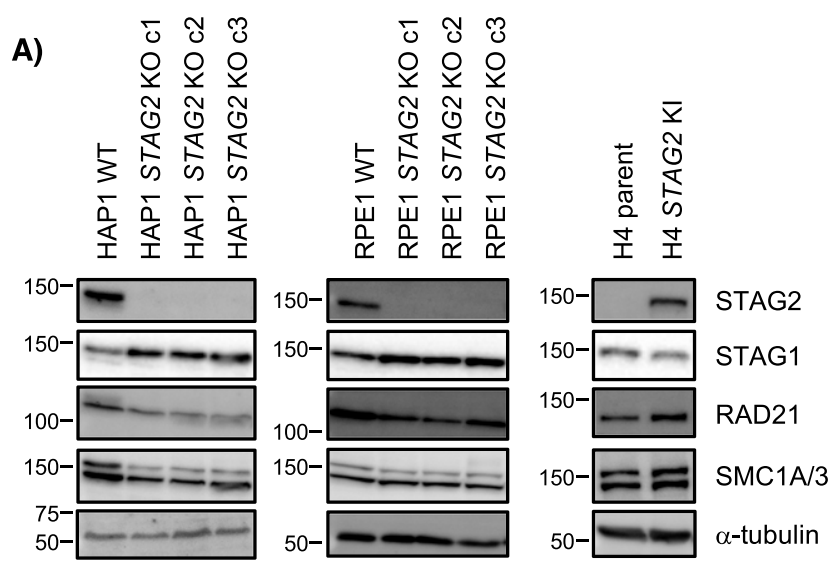

C)

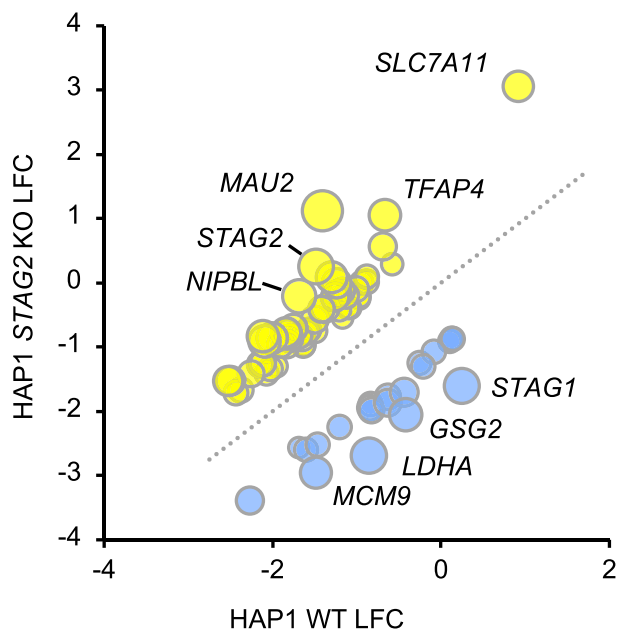

E)

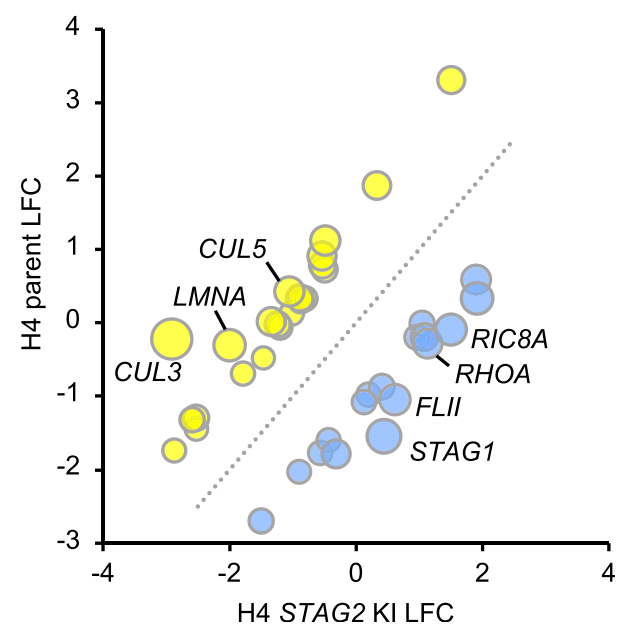

B)
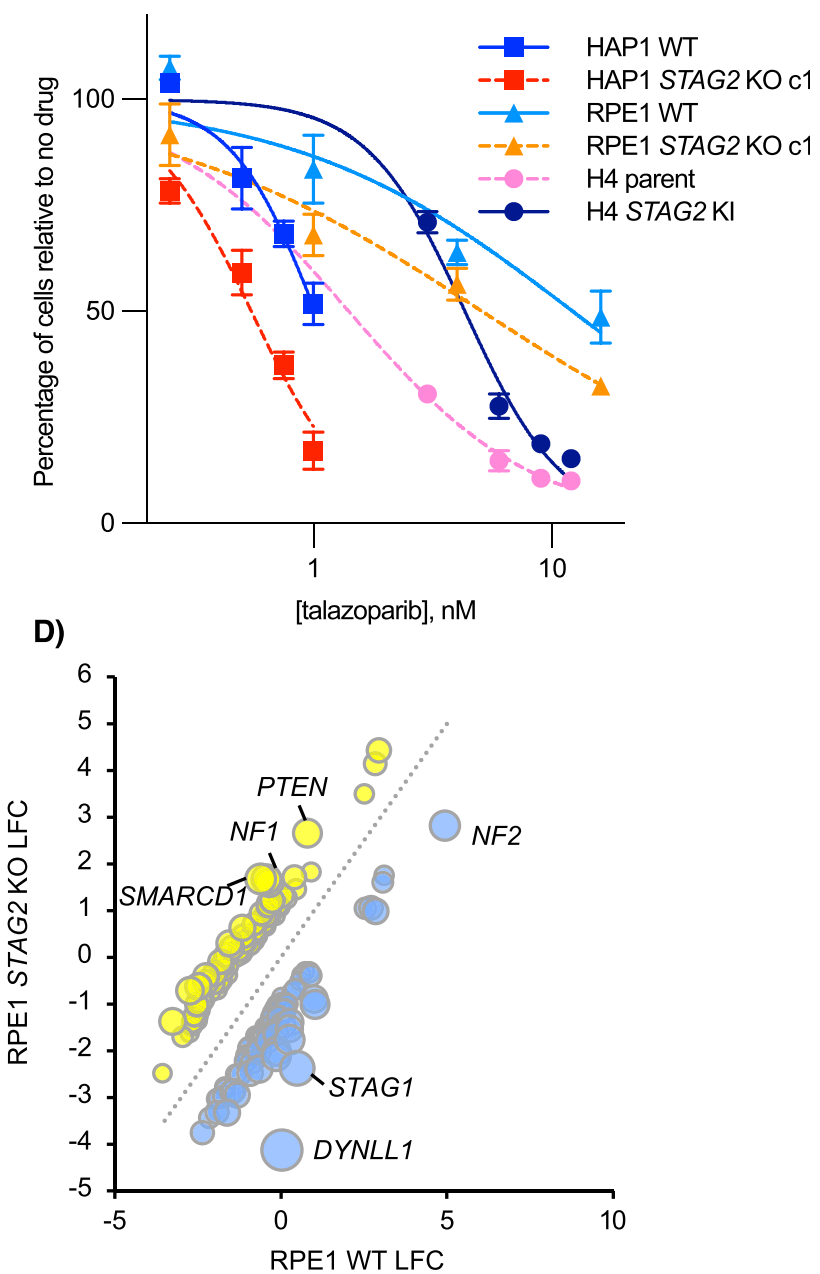

F)

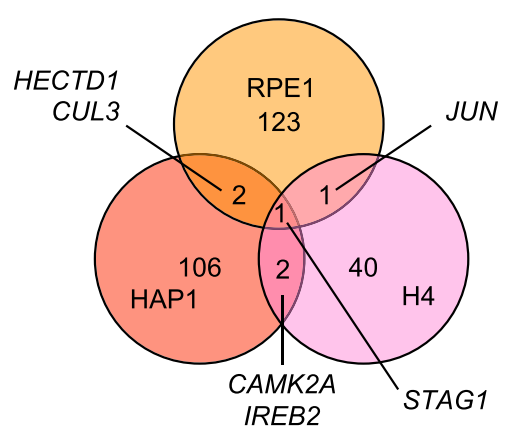

Figure 1. Whole-genome CRISPR-Cas9 knockout screen in cell lines with and without STAG2.

(A) Western blots of STAG2+ and STAG2- clones in three different backgrounds. Note that whereas HAP1 and RPE1 parents contain wild-type STAG2, H4 glioblastoma parent cells contain an endogenous STAG2 insertion that leads to protein truncation which is corrected in the knock-in (KI) cell line. (B) Effect of the PARP inhibitor talazoparib on select STAG2+ and STAG2- cells in three different backgrounds. Each line was normalized to a no drug control. Data were fitted with a non-linear IC ${ }_{50}$ Curve in GraphPad Prism. (C, D, E) Comparison of STAG2+ $\log _{2}$-fold change scores and STAG2- $\log _{2}$-fold change scores in (C) HAP1, (D) RPE1, and (E) H4 backgrounds at FDR < 0.2 . Candidate negative genetic interactions are in blue and candidate positive genetic interactions are in yellow. (F) Overlap of candidate STAG2 negative genetic interactions at a cut-off of $\mathrm{qGI}<-0.6$ for HAP1 and residual $<-1$ for RPE1 and H4. \% overlap at this cut-off is $2.18 \%$. 
Hill et al, 2016; Waldman, 2020), we performed genome-wide CRISPRCas9 knockout screens in three different genetic backgrounds to look for STAG2-dependent genetic interactions that could provide new insights into cohesin-STAG2 function and/or STAG2-based therapeutic strategies. Our screens confirmed STAG1 as the sole, strong context-independent negative genetic interaction for STAG2. We also found a previously unknown genetic interaction between STAG2 and an iron regulatory gene and a unique STAG2-dependent context in which the cohesin loading complex is no longer needed for cell viability. Collectively, our results highlight the multifunctional nature of cohesin-STAG2 and suggest that STAG1 is the only context-independent "loss-of-function" therapeutic target.

\section{Results}

\section{CRISPR-Cas9 screening of STAG2-positive and STAG2-negative cell lines}

To screen for genetic interactions with STAG2, we first obtained a series of STAG2-positive (STAG2+) and STAG2-negative (STAG2-) isogenic clones in three distinct cell line backgrounds. For HAP1 and RPE1 lines, we generated STAG2 KO clones using one of two single guide RNAs (sgRNA) that targeted STAG2 exonic DNA (Fig S1A and Table S1). Both HAP1 and RPE1 lines have been screened with CRISPR-Cas9 previously (Hart et al, 2015, 2017; Brown et al, 2019; Aregger et al, 2020), which we felt would be helpful in analyzing results from our isogenic STAG2 screens. Knockout of STAG2 in these two lines produced mainly small indels and coding frameshifts that led to early protein truncation (Table S1). We also obtained $\mathrm{H} 4$ parent cells that contain an endogenous truncating STAG2 mutation that has been corrected ex vivo in the H4 STAG2 knock-in (KI) line. These $\mathrm{H} 4$ lines have been described previously (Solomon et al, 2011) and were chosen because the parent represented a context in which the STAG2 mutation occurred and was adapted to in a tumour rather than being CRISPR generated. Western blots of all three cell line contexts confirmed the absence of STAG2 protein in STAG2- lines (Fig 1A). Our STAG2- lines also often showed compensatory up-regulation of STAG1 at the protein level (Fig 1A), a phenomenon that has been reported previously with RNAi (Kong et al, 2014).

As our HAP1 and RPE1 STAG2 KO cell lines were newly derived, we sought to further characterize them before performing whole-genome screening. To do this, we subjected parent and derived lines to a panel of 11 genotoxic and chemical stresses, some of which had shown STAG2-dependent responses previously (McLellan et al, 2012; Bailey et al, 2014; Mondal et al, 2019). Both HAP1 and RPE1 cells showed several STAG2-dependent responses, but these were often cell background-specific (Fig S1B). Only poly (ADP-ribose) polymerase (PARP) inhibitors showed a higher sensitivity in STAG2-depleted cells across all backgrounds (Figs 1B and S1B) demonstrating that our HAP1 and RPE1 STAG2 KO cell lines aligned with previous reports where STAG2 status was found to contribute to PARP inhibitor sensitivity (McLellan et al, 2012; Bailey et al, 2014; Mondal et al, 2019).

After genetic and chemical characterization, we undertook genomewide CRISPR-Cas9 knockout screens of STAG2+ and STAG2- lines in all three cell backgrounds using the Toronto Knockout (TKO) V3 CRISPR library (Hart et al, 2017; Aregger et al, 2019) (Fig S1C). All screens showed dropout of a reference set of core essential genes compared with a reference set of non-essential genes (Fig S1D and Table S2) (Hart et al, 2014, 2017). In addition, there was generally good correlation of $\log _{2}$ (aggregate sgRNA fold-change of end/initial reference time points) between different screens (Fig S1E). STAG2-dependent genetic interaction scores were calculated for each cell line background (see the Materials and Methods section; Tables S3-5) and candidate negative and positive genetic interactions at FDR $<0.2$ for each screen are shown in Fig $1 \mathrm{C}-\mathrm{E}$. Genetic interaction overlap between the three cell line backgrounds is shown with cut-offs for genetic interaction strength (Fig 1F) and statistical significance (Fig S1F). In both cases, only STAG1 shared a negative interaction in all three lines (Figs 1F and S1F) and few negative genetic interactions were observed with STAG2 across multiple genetic backgrounds, suggesting that the loss of STAG2 is highly buffered by STAG1 (Figs 1F and S1F).

\section{STAG1/STAG2 is a context-independent paralogous synthetic lethal interaction}

STAG1/STAG2 is an example of paralogous synthetic lethality where the two genes involved are two interchangeable subunits in an essential protein complex. In addition to our primary screens (Fig 1C-E), STAG1 and STAG2 have also recently been identified as a negative genetic interaction in several other backgrounds, including both H4 and RPE1 TP53 knockouts (Benedetti et al, 2017; van der Lelij et al, 2017, 2020; Liu et al, 2018; Mondal et al, 2019; Viny et al, 2019). To study the mechanism of this interaction further, we used CRISPR interference (CRISPRi) to knockdown STAG1 in wild-type or STAG2 KO lines (Fig S2A). Consistent with our primary screens, knockdown of STAG1 resulted in a preferential decrease in cell growth only in STAG2-deficient lines in all cell backgrounds (Fig 2A-C). Previous studies have shown that cells depleted of both STAG1 and STAG2 have a variety of mitotic phenotypes including defective sister chromatid cohesion, defective metaphase alignment and an increased mitotic index (van der Lelij et al, 2017, 2020; Liu et al, 2018). Although cell cycle distribution changes varied in our STAG2-deficient/STAG1-depleted backgrounds (Fig S2B), knockdown of STAG1 in STAG2- cell lines always resulted in an increased mitotic index as measured by an increase in phospho-serine 10 Histone $\mathrm{H3}$-positive ( $\mathrm{pH} 3+$ ) cells (Figs 2D-F and S2B). This suggests a shared mechanism for the STAG1/ STAG2 interaction regardless of cell background. Interestingly, HAP1 cells depleted of both STAG components showed a much higher increase in sub-G1 cells than the other cell lines (Fig S2B) which we speculate could be due to mitotic defects and/or TP53 mutations present in this background (Haarhuis et al, 2013; Yaguchi et al, 2018).

To determine whether the dependence of STAG2-mutated cells on STAG1 was a generalizable interaction beyond our three paired backgrounds, we examined the AVANA CRISPR Cancer Dependency Map (DepMap) dataset (Tsherniak et al, 2017; Behan et al, 2019). These data clearly showed a lower STAG1 gene effect score in cell lines with deleterious or damaging STAG2 mutations compared with those containing wild-type or other STAG2 mutations (Fig S2C). This suggests that, compared with STAG2 + cells, STAG2- cells are more dependent on STAG1 on a large, context-independent scale, a finding 
A)

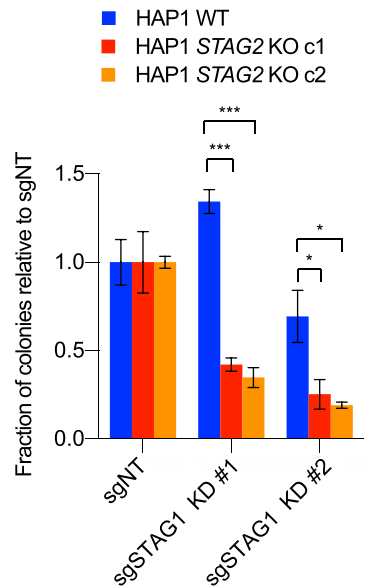

D)

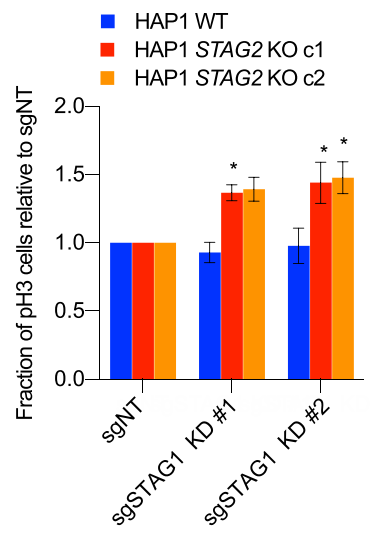

B)

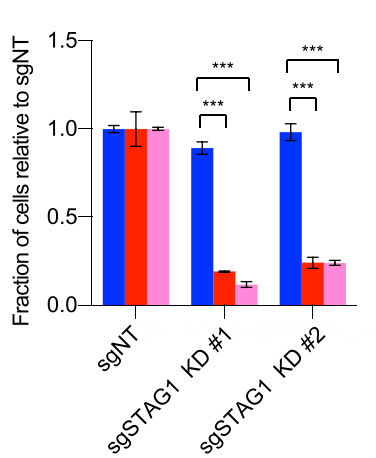

E)

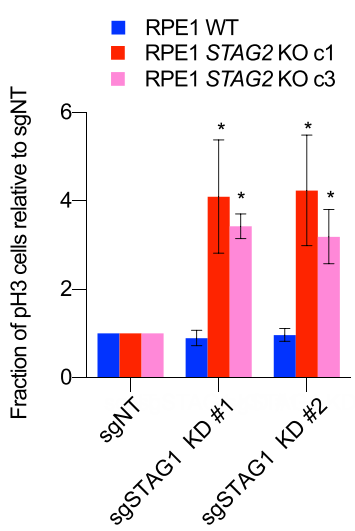

- RPE1 STAG2 KO C1

- RPE1 STAG2 KO c3
C)

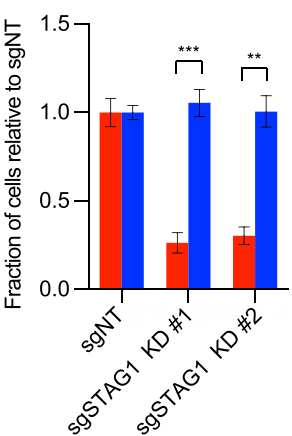

F)

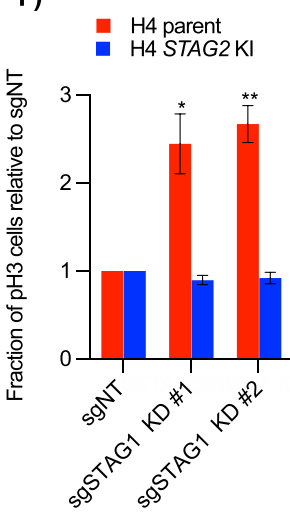

Figure 2. STAG1 knockdown in STAG2 KO cells. (A, B, C) Cell growth after infection of non-targeting or STAG1 knockdown sgRNAs in (A) HAP1, (B) RPE1, or (C) H4 cell lines stably expressing dCas9-KRAB. For the HAP1 lines, proliferation was determined by clonogenic assay. For H4 glioblastoma and RPE1 cells, cell growth was determined by nuclei counting. (D, E, F) Relative number of mitotic cells as analyzed by phosphoSer10 Histone $\mathrm{H} 3(\mathrm{pH} 3)$ staining and flow cytometry. Data in (D, E, F) represent the average of three independent experiments. ${ }^{*} P<0.05,{ }^{* *} P<0.005$, ${ }^{* * *} P<0.0005$ in a either a two-tailed, Welch's unpaired $(A, B, C)$ or a one-tailed, paired $(D, E, F)$ $t$ test. consistent with previous knockdown of STAG1 in bladder cancer and Ewing's cell line panels (van der Lelij et al, 2017).

\section{A genetic interaction between STAG2 and the iron regulatory gene IREB2}

Whereas STAG1 was the only negative genetic interaction shared in all three cell backgrounds, several other gene candidates were found in two out of three lines and we wondered if we could learn more about STAG2 function and genetic interaction context-dependency by further characterizing one of these, namely, the STAG2/IREB2 interaction observed in the cancer-derived HAP1 leukemia and H4 glioblastoma cell lines.

In normal cells, iron levels must be tightly controlled (Fig 3A). Sufficient iron levels are needed for cell essential processes such as metabolism and DNA synthesis; however, excess iron can promote the formation of free radicals that contribute to cellular damage and death (Katsarou \& Pantopoulos, 2020). IREB2 (protein name IRP2) and its paralog ACO1 control the iron regulatory response by directly responding to changes in intracellular iron levels. As iron levels increase, the IRP2 protein is degraded, whereas ACO1 protein levels remain unaffected, but the protein undergoes a conformational change to an aconitase (Fig 3A) (Kühn, 2015). Conversely, when iron levels in the cell are low, both iron response protein (IRP) proteins can bind iron-responsive element (IRE)- containing mRNAs which may either increase in abundance or decrease expression depending on IRE locations and mRNA function (Kühn, 2015). Interestingly, increases in intracellular iron have been observed in many different tumour types (Torti \& Torti, 2013), and we wondered if there might be a connection between STAG2 loss-offunction and iron dysregulation given the potential STAG2/IREB2 genetic interaction in HAP1 and $\mathrm{H} 4$ cells, the two transformed cell line contexts (Fig 1F).

Cell growth assays confirmed that knockout of IREB2 was more detrimental in STAG2- cells than STAG2 + cells in both the HAP1 and $\mathrm{H} 4$ contexts (Figs $3 \mathrm{~B}-\mathrm{D}$ and $\mathrm{S} 3 \mathrm{~A}-\mathrm{D}$ ). The same negative genetic interaction was also observed in a 42 MGBA parent/STAG2 KI cell line pair which, like the $\mathrm{H} 4$ context, contains a naturally occurring truncating mutation in the parent line which is corrected in the $\mathrm{KI}$ line (Solomon et al, 2011), as well as a HCT116 WT/STAG2 KO cell line pair (Fig S3E-G). Interestingly, we did not see a negative genetic interaction between STAG2 and IREB2 in RPE1 cell lines (Figs 3E and $\mathrm{S} 3 \mathrm{H}$ ), consistent with the primary screen data. We note that in all our backgrounds the STAG2/IREB2 interaction appears more moderate than STAG1/STAG2. Furthermore, DepMap screening data also showed more modest STAG2-dependent differences in the IREB2 gene effect score in various lineages (Fig S3I). Therefore, we conclude that STAG2 / IREB2 is a modest negative genetic interaction in four out of five of our STAG2 isogenic contexts. 
A)

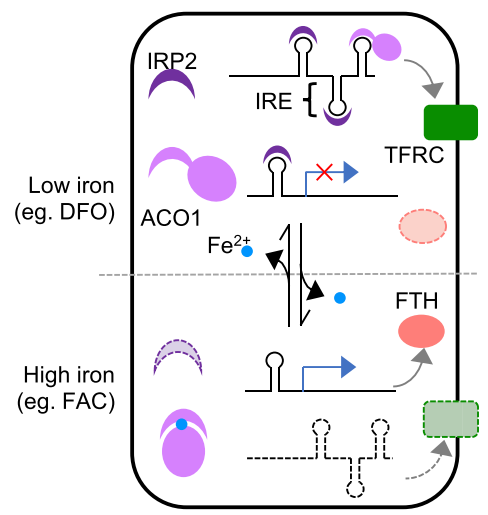

D)

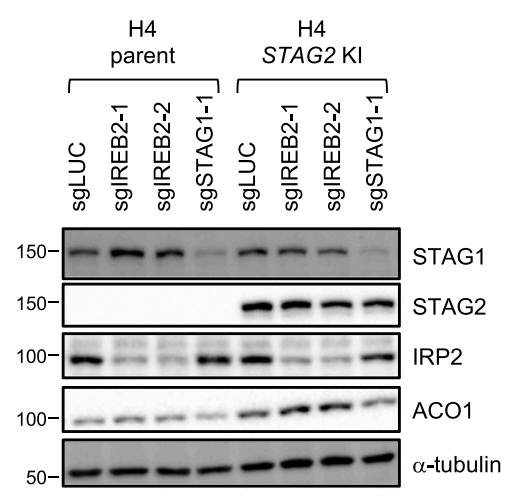

G)

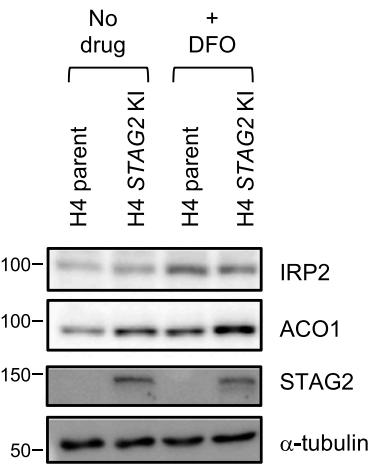

B)

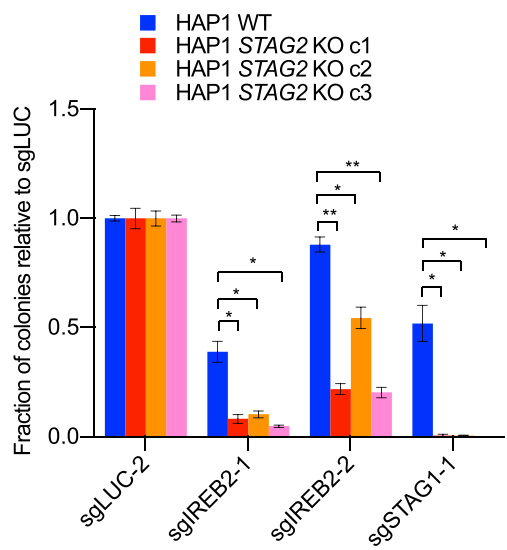

C)

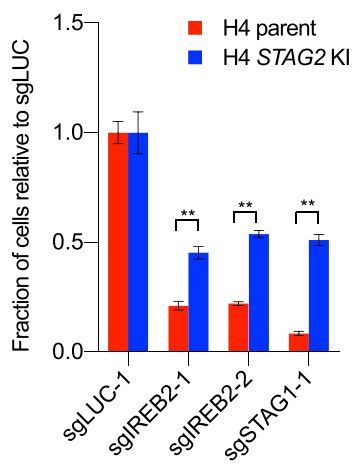

E)

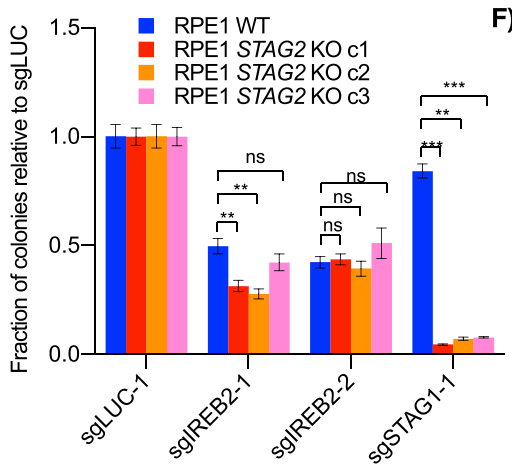

H)
"
ธ ชู ชู

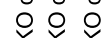

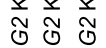

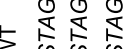

占的的

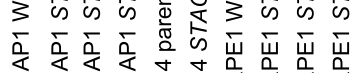

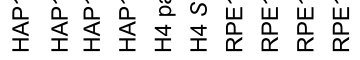

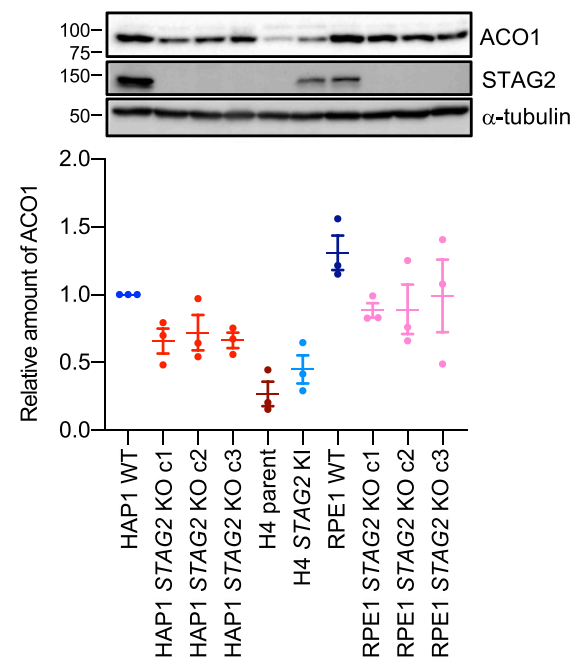

l)
F)
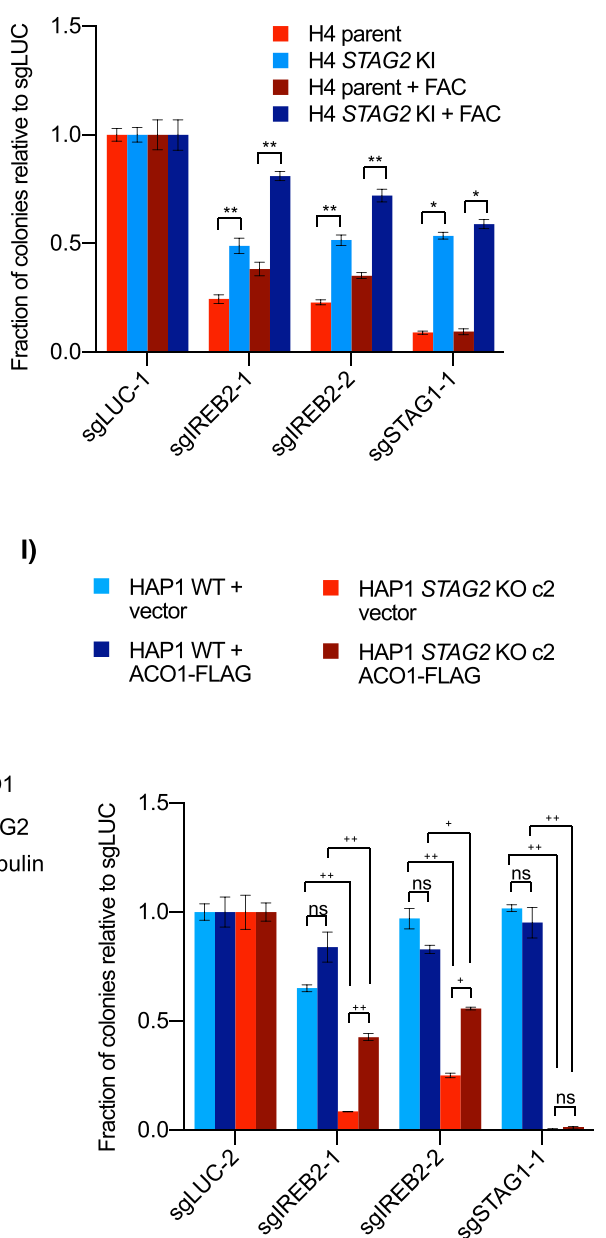

Figure 3. Genetic interaction between STAG2 and IREB2.

(A) Schematic of ACO1 and IRP2 function in cells. In low-iron conditions (e.g., DFO), ACO1 is in an RNA-binding form and IRP2 (the gene product of IREB2) is stabilized. Both bind iron responsive elements in target mRNAs and either stabilize them (as in the case of the iron importer TFRC) or block their translation (as with the ferritin component FTH). In high iron conditions (e.g., FAC), the IRP proteins are not needed and ACO1 is converted to an aconitase form, whereas IRP2 is degraded which destabilizes the mRNA of TFRC and allows translation of FTH. (B) Growth of HAP1 WT and STAG2 KO cells after transfection of control or IREB2 sgRNA as determined by clonogenic assay. (C) Interaction between STAG2 and IREB2 KO in H4. Cells were infected with control and IREB2 sgRNA before being normalized and re-plated for growth 
As IREB2 is a master iron regulator in the cell, we tested whether adding excess iron affected the interaction of STAG2 and IREB2. We found that, compared with standard media, there was no change in the strength of the negative genetic interaction between IREB2 and STAG2 when ferric ammonium citrate (FAC) was added (Fig 3F) although iron addition did partially rescue proliferation defects after IREB2 knockout in both $\mathrm{H} 4$ parent and STAG2 KI lines indicating FAC treatment was partially effective. The genetic interaction was also unaffected when a low level of deferoxamine (DFO) was added (Fig S3J). DFO is an iron chelator that has been used clinically to treat excess iron burden (Kontoghiorghe \& Kontoghiorghes, 2016).

The above results suggest that STAG2- cells are not more dependent on IREB2 for proliferation because of an upstream ironrelated stress or a down-stream iron-dependent dysregulation. We next considered how STAG2 loss-of-function affected IRP IRP2 and its paralog ACO1. Western blots of STAG2 + and STAG2- cells showed similar levels of IRP2 even after altering IRP2 levels with DFO (Fig 3G). There was, however, a detectable decrease in the IRP2 paralog ACO1 in the STAG2- lines compared with the STAG2+ lines across multiple conditions and backgrounds (Fig 3D, G, and H). Quantification of ACO1 levels in HAP1, H4, and RPE1 STAG2+ / - backgrounds showed absolute levels of ACO1 differed across the three backgrounds (Fig 3H). Interestingly, RPE1 cells had the highest ACO1 levels and also did not demonstrate a STAG2/IREB2-negative genetic interaction. As the DepMap CRISPR screening data suggested that lower ACO1 levels can affect the proliferative response of IREB2 $\mathrm{KO}$, at least up to a certain point (Fig S3K), we speculated that there was a threshold expression below which levels of ACO1 are not sufficient to fully buffer loss of IREB2.

To determine if higher ACO1 levels affect the strength of the STAG2/IREB2 interaction, we co-expressed ACO1-FLAG and IREB2 sgRNAs in HAP1 STAG2+ / - cells. Exogenous ACO1 expression partially rescued the proliferative defect in STAG2 KO cells, but did not have a significant effect in WT cells (Figs 31 and $\mathrm{S} 3 \mathrm{~L}$ ). This suggests that increasing expression of ACO1 can mitigate the strength of the STAG2/IREB2 interaction and provides an explanation for why RPE1 cells, which have higher endogenous levels of ACO1, do not show a significant STAG2/IREB2 interaction. It also suggests that decreases in ACO1 expression in STAG2- cells can make them more susceptible to loss of the other IRP paralog when absolute ACO1 levels are below a threshold.

To further investigate the effects of STAG2 loss on iron regulation, we treated STAG2+ and STAG2- cell lines with the iron chelator DFO. Across multiple backgrounds, we found no consistent STAG2-dependent proliferation response to DFO after either acute or chronic treatment with the chelator, providing no obvious link between STAG2 status and growth in low iron conditions (Figs 4A-C and S4A-C). We also looked at levels of two IRP targets: TFRC/TFR1, an iron importer that increases in low iron and ferritin heavy chain (FTH), a subunit of the ferritin storage complex, that increases in high iron (Torti \& Torti, 2013). Western blots showed increased stabilization of FTH in STAG2cells compared with STAG2+ cells after iron addition, but no STAG2dependent difference in TFRC (Figs 4D and S4D). Although ACO1 and IREB2 are paralogs and largely functionally redundant, molecular studies have shown preferential binding affinities to several IRE sequences and target regulation which may partly explain this result (Wang et al, 2007; Kühn, 2015). As well, IREB2 is thought to be the more important regulator for iron response in mice and in human cells (Meyron-Holtz et al, 2004; Wang et al, 2007), which may make ACO1-dependent responses in STAG2 KO cells more difficult to observe when IREB2 is present. We therefore cannot confirm a link between STAG2 KO and iron response and suggest the mechanism behind the STAG2/IREB2 genetic interaction occurs at the paralog level in isogenic lines. In STAG2 KO lines, IRP2 can likely compensate for decreased ACO1 levels. It is only after IREB2 is depleted and ACO1 becomes the dominant paralog that cells are more susceptible to STAG2-dependent regulation.

\section{A unique STAG2-dependent positive genetic interaction with cohesin loaders}

Although the original goal of our STAG2 screen was to look for negative genetic interactions common to multiple backgrounds, we also observed an unexpected positive candidate interaction in the HAP1 primary screen between STAG2 and both subunits of the cohesin loader (Fig 1C). In humans, the heterodimeric cohesin loader complex, composed of NIPBL and MAU2, loads cohesin onto DNA, typically in regions associated with promoters and active transcription (Liu et al, 2009; Newkirk et al, 2017). Although the cohesin loader genes are essential in Saccharomyces cerevisiae, more recent studies in vitro and in mammalian cells have suggested a role for both STAG1 and STAG2 in the chromatin localization of cohesin, either through binding of the insulator protein, CTCF, or by binding DNA directly (Rubio et al, 2008; Xiao et al, 2011; Bisht et al, 2013; Countryman et al, 2018; Kojic et al, 2018; Pherson et al, 2019). It was surprising then, that NIPBL and MAU2 were identified as strong candidate positive genetic interactions in the HAP1 background (Fig 1C). This would suggest that after STAG2 is lost, the loaders become less essential in this background.

To verify the genetic interaction, we used clonogenic assays and confirmed that knockout of either MAU2 or NIPBL resulted in a positive interaction with STAG2 KO in HAP1 but not RPE1 or H4 cell line backgrounds (Figs 5A and B and S5A and B). Using HAP1 STAG1 clonal knockout cell lines, we observed a similar suppressive interaction between MAU2 and STAG1 (Fig S5C). Double transfection of SgRNAs for STAG2 and MAU2 into HAP1 WT cells also resulted in a positive interaction (Fig S5D). Together, these data confirmed a

assessment. Cell number was determined by nuclei counting. (D) Levels of iron regulatory proteins in H4 parent and STAG2 KI cell lines including the IREB2 gene product IRP2 after infection with IREB2 and STAG1 sgRNAs. (E) Effect of sgIREB2 KO on the growth RPE1 WT and STAG2 KO cells as determined by nuclei counting. (F) Effect of ferric ammonium citrate on STAG2/IREB2 genetic interaction. Cells were infected and normalized as in (C) before $50 \mu \mathrm{M}$ ferric ammonium citrate was added $\sim 24 \mathrm{~h}$ after replating. (G) Levels of iron regulatory proteins IRP2 and ACO1 in H4 parent and STAG2 KI cells after either no treatment or treatment with $100 \mu \mathrm{M} \mathrm{DFO} \mathrm{for} 16 \mathrm{~h}$. (H) (Top) Western blot of ACO1 levels across both STAG2+ and STAG2- cell lines in HAP1, H4 and RPE1 backgrounds. (Bottom) Amount of ACO1 as compared with HAP1 WT in three independent lysates. (I) Clonogenic growth of HAP1 WT and STAG2 KO cells after co-transfection of either vector (pCDNA3.1) or ACO1-FLAG plasmids and IREB2 sgRNAs. nS, not significant, ${ }^{*} P<0.05,{ }^{* *} P<0.005,{ }^{* *} P<0.0005$ in a Welch's two-tailed $t$ test. ns, not significant, ${ }^{+} P<0.005,{ }^{++} P<0.0005$ in a one-way ANOVA plus TUKEY. 
A)

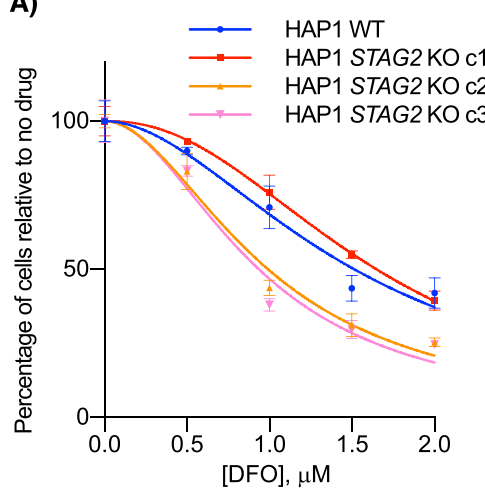

C)

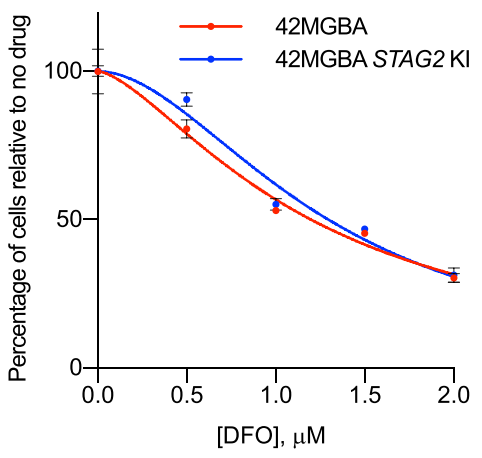

B)

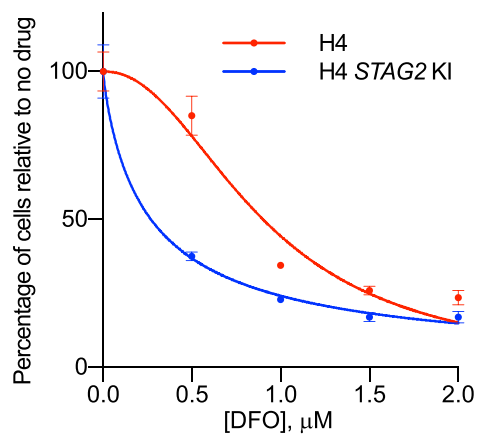

D)

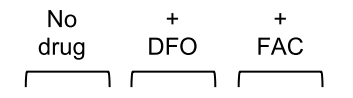

Figure 4. STAG2 status and iron response. (A, B, C) Cell growth of (A) HAP1, (B) H4 and (C) 42 MGBA STAG2+/- cells in the presence of the iron chelator DFO. (D) Levels of iron-related proteins including IRP targets TFRC and FTH in $\mathrm{H} 4$ parent and STAG2 KI cells after no treatment or treatment with $100 \mu \mathrm{M}$ DFO or $300 \mu \mathrm{M}$ ferric ammonium citrate. context-dependent interaction between STAG1/2 and the cohesin loaders in HAP1 cells.

Further analysis of the interaction showed no obvious cell cycle changes in either WT or STAG2 KO cells after the MAU2 gene was deleted (Fig S5E and F). Chromatin fractionation also showed that all three core ring components (SMC1A, SMC3, and RAD21) had no detectable differences in bulk chromatin binding after loader depletion (Fig 5C). Although a previous study using NIPBL+/- MEF also did not observe any decrease of core cohesin components on chromatin after fractionation (Remeseiro et al, 2013), that same study and others have seen a decrease in core components in both heterozygous MEFs and HAP1 cohesin loader knockouts using ChIP and, in the case of HAP1 cells, immunofluorescence (Remeseiro et al, 2013; Haarhuis et al, 2017; Newkirk et al, 2017). Therefore, we speculate that chromatin fractionation may not be sensitive enough or our experiment may not have been long enough (only $7 \mathrm{~d}$ posttransfection of sgRNAs) to observe cohesin changes at the global chromatin level. Interestingly, knockout of MAU2 in HAP1 WT cells resulted in an increase in STAG1 in lysates compared with control, a similar effect to that observed at the RNA level (Rollins et al, 2004; Kawauchi et al, 2009; Liu et al, 2009). Surprisingly, chromatin fractionations showed this increased STAG1 was mainly in the soluble fraction rather than on chromatin and seemed unlikely to be physically associated with the chromatin-bound core cohesin subunits. This up-regulation of STAG1 after MAU2 deletion was also seen in HAP1 STAG2 KO cells (Fig 5C).

Finding a context where cohesin loaders were non-essential was unexpected and we therefore wondered whether there were any other contexts besides HAP1 STAG2 KO cells where cohesin loaders were not essential. Gene dependency scores in the DepMap screening dataset showed that depletion of the cohesin loader genes had a similar range of dependency scores to many of the other accessory factors of cohesin, especially when compared with the three essential core ring components (Fig S5G). Consistent with the loaders working as a heterodimer, scores of the two loader genes correlated well across all cell lines, with a very small fraction of cell lines showing positive gene dependency scores after depletion of either loader (Fig S5H). This suggests that there are other cell line contexts, albeit a very small number of them, in which loss of a cohesin loader gene showed little or no fitness defect.

Correlations in cell line essentiality profiles can suggest functional relationships between genes (Pan et al, 2018; Kim et al, 2019), so we expanded our analysis of the DepMap dataset and found three other genes, PAXIP1 (protein name PTIP), its binding partner PAGR1 (also known as PA1) and PRR12, whose gene dependency score profiles correlated well with the two loader genes and STAG1/2 
A)

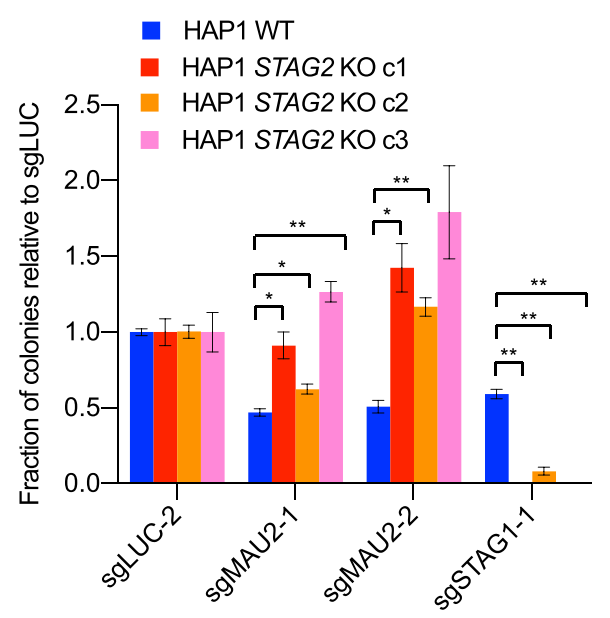

C)

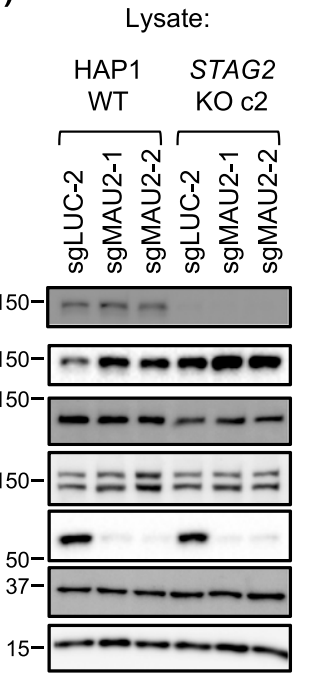

B)

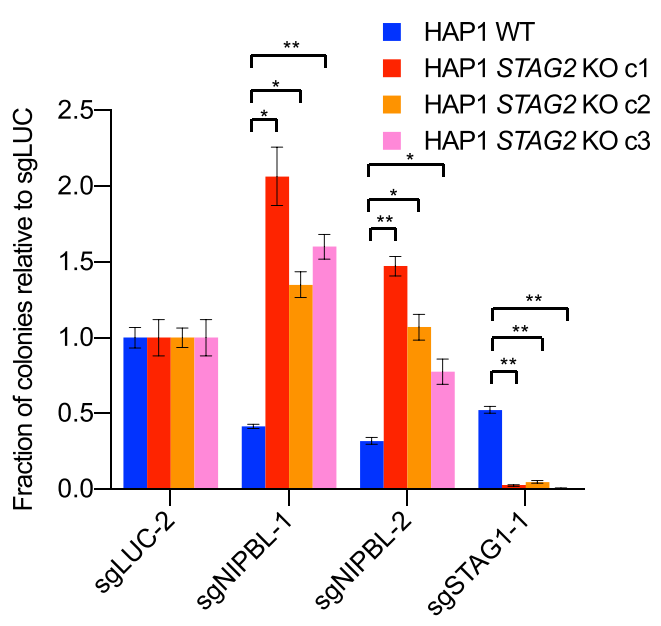

D)

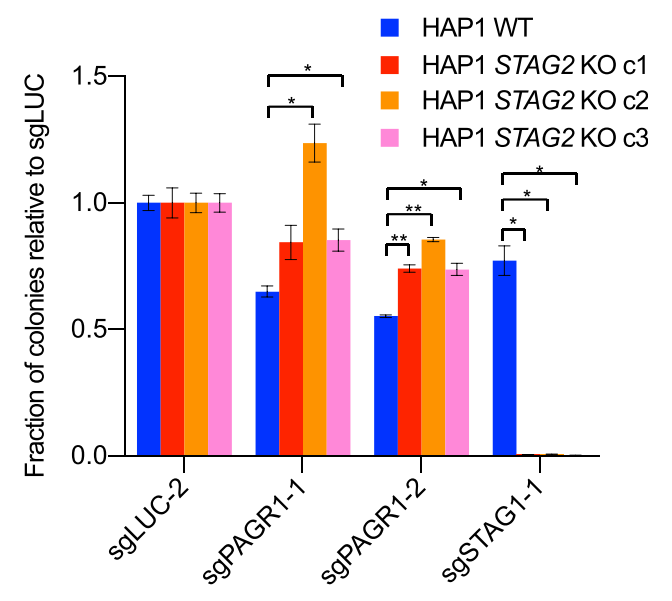

Figure 5. Knockout of cohesin loaders in HAP1 STAG2 KO cells.

(A, B, D) Clonogenic growth of HAP1 wild-type and STAG2 KO cells after transfection of sgRNAs for MAU2 (A), NIPBL (B) or PAGR1 (D). (C) Levels of cohesin proteins in lysates and soluble and chromatin fractions of HAP1 WT and STAG2 KO cells after transfection with control and MAU2 sgRNAs. ${ }^{*} P<0.05,{ }^{* *} P<0.005$.

(Fig S5I and J). Both PAXIP1 and PAGR1 were candidate positive interactions in the HAP1 primary screen (qGIs of 0.882 and 0.847 , respectively) and we validated STAG2/PAGR1 as a positive interaction using clonogenic assays (Fig 5D).

Taken together, we found the DepMap CRISPR dataset supports the possibility of a small subset of genetic contexts, like HAP1 STAG2 $\mathrm{KO}$, where there is less dependence on cohesin loaders for cell fitness.

\section{Discussion}

Genetic interaction profiles obtained by genome-scale CRISPR knockout screens can have functional and therapeutic implications. In recent years, however, more studies have recognized that many genetic interactions are influenced by genetic and environmental factors including cell line background, epigenetic profiles and various endogenous and exogenous stresses (Ryan et al, 2018; Shen \& Ideker, 2018). In this study, we used CRIPSRCas9 whole-genome screening to investigate potential genetic interactions of STAG2, a member of the cohesin complex that has a high rate of loss in certain types of cancer. We performed isogenic screens in three different cell backgrounds and found several points of comparison with the DepMap screening initiative, but little overlap of genetic interactions among all three backgrounds. Only one strong and conserved negative genetic interaction was found between STAG2 and its paralog STAG1, a finding consistent with a recent isogenic STAG2 screen in KBM7 cells (van der Lelij et al, 2020).

In a combinatorial CRISPR study investigating pairwise genetic interactions of 73 genes in three cell lines, (Shen et al, 2017) found that $\sim 10 \%$ of the observed negative genetic interactions were conserved in more than one cell line, but none overlapped in all three cell lines (Shen et al, 2017). In contrast, only 2\% of STAG2dependent negative genetic interactions overlapped in two or more cell line backgrounds in our screens, with one hit common in all 
three backgrounds; that is, STAG1 (Figs 1F and S1F). One reason for this lower overlap could be technical. Although a combinatorial CRISPR screen introduces both sgRNAs into a population of cells at the same time, our screens were done in lines isolated from a from a single colony where both STAG2-independent clonogenic effects and background-specific outgrowth adaptation could significantly decrease genetic interaction overlap.

Another reason for the lower overlap observed in our screens could be the function of STAG2 itself. The $10 \%$ negative genetic interaction overlap found by Shen et al represents an average across the 73 genes tested and it may be that certain genes simply have larger numbers of context-independent genetic interactions. For example, a recent study of PARP inhibitor-dependent gene sensitivities found that $>47 \%$ reproduced in more than one cell line background; however, an independent study using an ATR inhibitor found only $9 \%$ of ATR inhibitor gene sensitivities reproduced in a second cell line (Zimmermann et al, 2018; Wang et al, 2019).

The characteristic(s) of a gene or genetic interaction that determine context-dependency are still largely unknown, but in the case of STAG2-cohesin, we offer three possible reasons for lower genetic interaction overlap between multiple contexts: (1) Although cohesin is multifunctional, not all cellular functions require the same amount of the complex. Studies in yeast and loader-deficient mammalian cells have suggested a "dosage-sensitive functional hierarchy" for cohesin where functions such as gene regulation are most sensitive to changes in cohesin levels, whereas essential mitotic functions such as sister chromatid cohesion require only a very small amount of the complex (Heidinger-Pauli et al, 2010; Newkirk et al, 2017). (2) The STAG2 paralog, STAG1, can likely buffer most essential cohesin functions after STAG2 loss. Although there is evidence for STAG2-specific functions in DNA repair and telomere recombination (Kong et al, 2014; Daniloski \& Smith, 2017), we did not observe consistent enrichment of genes in these pathways in our primary screens under the conditions tested. (3) Other unbuffered STAG2-only functions appear cell type specific. STAG2 is known to regulate cell type-specific transcription and contribute to HSPC "stemness" (Mullenders et al, 2015; Galeev et al, 2016; Kojic et al, 2018), suggesting that there may be a higher number of overlapping genetic interactions among cell backgrounds with shared differentiation programs and/or transcriptional profiles.

Both STAG1/STAG2 and IREB2/ACO1 are examples of paralogous synthetic lethal pairs (Smith et al, 2006; Benedetti et al, 2017; van der Lelij et al, 2017, 2020; Liu et al, 2018; Viny et al, 2019). Although we confirmed STAG1 as a context-independent paralogous synthetic lethal interaction of STAG2, we also uncovered buffering between the IRP paralogs as a key factor underlying the mechanism of the STAG2/IREB2 genetic interaction. Although the STAG2/IREB2 genetic interaction was initially chosen because of the relationship between iron dysregulation and cancer, we found no evidence that iron played a role in the STAG2-negative genetic interaction with IREB2 (Fig 3F). Instead, we linked proliferation defects in multiple isogenic contexts to lower levels of ACO1 in STAG2 KO cells. Although more complex than the STAG1/STAG2 paralogous synthetic lethal interaction, this mechanism also helps explain the context-dependency of STAG2/IREB2 as buffering of IREB2 loss is only needed at low expression levels of ACO1 and ACO1 levels are only partly controlled by STAG2.

Although paralogous synthetic lethal pairs like STAG1/STAG2 and IREB2/ACO1 are interesting biologically, how the large therapeutic window of these paralogous synthetic lethal interactions can be translated into potential cancer therapeutics is an open question. Although paralogs can be easily distinguished genetically with RNAi or CRISPR, they are more difficult to distinguish pharmacologically. STAG1 and STAG2, for example, are more than 85\% similar at the protein level which will make the development of paralog-specific inhibitors, especially for non-enzymatic proteins, very difficult.

The original goal of our STAG2 screen was to look for genetic interactions common to multiple backgrounds; however, we also observed and confirmed a highly unexpected positive interaction in the HAP1 background between STAG2 and both subunits of the cohesin loader (Figs 5 and S5). Our analysis suggested there may be some functional relationship between the cohesin loaders, STAG2 and the multifunctional PTIP/PA1 complex (Fig S5J). Exactly which function of PTIP/PA1 may be important with respect to STAG2 and cohesin function is at this point unknown. PTIP has been shown to function in both histone methylation and stalled replication fork protection through its interaction with the MLL3/4 complex and to promote DNA damage response with PA1 in a RNF8-dependent manner (Patel et al, 2007; Cho et al, 2007; Wu et al, 2009; Chaudhuri et al, 2016); however, these factors do not show an obvious correlation with the cohesin loader or STAG2 in the DepMap data (Fig S5I). The PTIP/PA1 complex also plays a role in class switch recombination where it promotes MLL3/4independent H3K4 methylation as well as long-range chromatin interactions (Schwab et al, 2011; Starnes et al, 2016). Furthermore, both PTIP/PA1 and cohesin loading localize mainly to open chromatin and transcriptionally active promoters, which also supports a functional association between these complexes (Lechner et al, 2000; Liu et al, 2009; Newkirk et al, 2017). Future studies that explore this relationship between cohesin loading and the PTIP/PA1 complex could provide insights into not just cohesin dynamics and function but also the developmental disease Cornelia de Lange Syndrome which is often characterized by heterozygous mutations in NIPBL (Sarogni et al, 2020).

Based on our screen results and those of others, it seems unlikely that a "loss-of-function" therapeutic target beyond STAG1 will be discovered that will work in all diverse cancer types with STAG2 loss. In the future, it may be of greater benefit to determine STAG2 dependencies in groups of cells lines that closely share a lineage, transcriptional program or a cohesin-related vulnerability. Alternatively, better overlap among STAG2-dependent genetic interactions might be achieved by screening for "gain-of-function" mutations in synthetic lethal partner genes or by using agents such as PARP inhibitors that have shown greater context-dependency across multiple STAG2-deficient and STAG2-mutated cell lines (McLellan et al, 2012; Yang et al, 2013; Bailey et al, 2014; lorio et al, 2016; Mondal et al, 2019). PARP inhibitors induce genomic stress by creating DNA lesions and inhibit DNA repair, a known cohesin function. 


\section{Materials and Methods}

\section{Plasmids, cell lines, and antibodies}

For generation of knockout lines, sgRNAs (Table S6) were cloned into pSpCas9-T2A-puro (\#62988; Addgene). The puromycin resistance gene caused variable puromycin resistance in RPE1-hTERT cells, so T2A-puro was swapped for T2A-Blast using EcoRI. For all subsequent sgRNA KO experiments, sgRNAs were cloned into LentiCRISPR V2 (\#52961; Addgene). An EF1 $\alpha$-FLAG-dCAS9-KRAB-T2A-Blast plasmid was used to express dCAS9-KRAB for all CRISPRi experiments. This construct was made by amplifying EF1 $\alpha$ from pCRISPRia (\#84832; Addgene), T2A-Blast from dCas9-VP64 Blast (\#61425; Addgene) and KRAB from Lenti-dCas9-KRAB-Blast (\#89567; Addgene) before sequentially cloning each product into pLV hUbC-dCas9-VP64-T2A-GFP (\#53192; Addgene) using Pacl/Xbal, Nhel/Agel, and Nhel, respectively. CRISPRi sgRNAs were cloned into pCRISPRia. sgRNAs were verified correct by sequencing before being used. ACO1-FLAG was from Genscript.

All cell lines were grown in 10\% FBS (Invitrogen) and incubated at $37^{\circ} \mathrm{C}$ and $5 \% \mathrm{CO}_{2}$. HAP1 cells were cultured in IMDM. $\mathrm{H} 4$ and $42 \mathrm{MGBA}$ cells (gift from T Waldman) and RPE1-hTERT cells were grown in DMEM. HCT116 WT and STAG2 KO (gift from T Waldman) were grown in McCoy's medium.

Antibodies used for Western blot were as follows: from Abcam, SMC1 (ab9262), SMC3 (ab9263), a-tubulin (DM1A) (ab7291), FTH (ab75972), MAU2/Scc4 (ab183033), GAPDH (ab9485), and HISTONE H3 (ab1791); from GeneTex, STAG1 (GTX129912) and ACO1 (GTX128976); from Millipore, RAD21 (05-908); from Novus, CAS9 (NBP2-36440); from Santa Cruz, STAG2 (sc81852), IRP2 (sc33682), and TFRC (sc65582); from SigmaAldrich, FLAG M2 (F1804); rat-anti-tubulin was clone YOL1/34. Secondary antibodies were either goat-anti-rabbit, goat-anti-mouse, or goat-anti-rat conjugated to HRP, Alexa Fluor 488 or Cy3 (Jackson Laboratories).

\section{Generation of clonal knockout lines}

For HAP1 and RPE1 STAG2 KO clones, parent cells were transfected with pSpCas9-T2A-Blast plasmid using XtremeGene 9 (Roche) according to manufacturer's instructions. The following day, transfected cells were selected for $\sim 3 \mathrm{~d}$ using Blasticidin (Sigma-Aldrich). For HAP1 KO lines, cells were then replated at single cell density in $10 \mathrm{~cm}$ plates. 10-14 d after plating, colonies were picked using cloning cylinders and transferred to a 96-well dish. For RPE1 cells, cells were plated at limiting dilution in 96-well plates. All clones were passaged until they reached $10 \mathrm{~cm}$ density. Clones were then sequenced for a mutation near the expected target site and any expected protein knockouts were confirmed by Western blot. All parent lines and STAG2 KO clones were checked for mycoplasma before being used. HAP1 clones were also stained with propidium iodide and compared with parent cells to determine ploidy.

\section{CRISPR-Cas9 knockout screens}

All CRISPR-Cas9 screens were performed as previously described (Aregger et al, 2019). Briefly, cells were infected with lentiviral TKOv3 library at an $\mathrm{MOI}$ of $\sim 0.3$ then selected the following day with puromycin $(1 \mu \mathrm{g} / \mathrm{ml}$ for $\mathrm{H} 4$ cells, $2 \mu \mathrm{g} / \mathrm{ml}$ for HAP1 cells and $8 \mu \mathrm{g} / \mathrm{ml}$ for RPE1 cells) for $48 \mathrm{~h}$ for HAP1 and $\mathrm{H} 4$ cells. For RPE1 lines, cells were selected for $72 \mathrm{~h}$ with replacement of selection media after 24 h. After selection (i.e., T0), the cells were replated in three replicates at 200-fold coverage of the library. Replicates were passaged every 3-4 d maintaining coverage of the sgRNA library and with three samples collected at T0 and all subsequent passages, until the infected population reached 20 doublings. Genomic DNA was purified from TO and end point samples using Promega Wizard Genomic DNA Purification kit according to manufacturer's instructions. For each sample, sgRNA inserts were amplified from $52.5 \mu$ g of genomic DNA by a two-step PCR reaction using primers harboring Illumina TruSeq adaptors with i5 and i7 barcodes. The sequencing libraries were gel purified and sequenced on a Illumina HiSeq 2500. For the HAP1 screen, each STAG2 KO clone was run as one replicate and the three replicates were combined for dropout scoring. For RPE1 (WT and STAG2 KO C1) and H4 glioblastoma (parent and STAG2 KI) screens, a midpoint (T18 for RPE1 and T14 for H4) was also lysed and run. For the HAP1 STAG2 KO screen, $\log _{2}$-fold change and genetic interaction (qGI) scores were processed and calculated as in Aregger et al (2020). For RPE1 T18 and H4 T24 screens, log 2 -fold change and genetic interaction residuals were similarly calculated but without correction for multiple wild-type screens.

\section{STAG1 knockdown experiments}

Cell lines were infected with the dCas9-KRAB construct. HAP1 and $\mathrm{H} 4$ cells were infected as in primary screens, but because of a significant growth lag, all RPE1 infections after the primary screen were performed as follows: infection with virus in 6\% FBS for 5-6 h followed by recovery in virus-free medium with $10 \%$ FBS for $18-19 \mathrm{~h}$. After $24 \mathrm{~h}$, all infected cells were selected with Blasticidin. 7-10 d after KRAB infection, cells were collected and infected with pCRISPRia constructs. After $48 \mathrm{~h}$ (H4) or $72 \mathrm{~h}$ (RPE1) of puromycin selection, cells plated in triplicate in 96-well plates were grown in drug-free medium for 3-5 d, before being fixed with paraformaldehyde, stained with Hoechst 33342 and nuclei counted using a Cellomics LTV machine. HAP1 cells were selected for $48 \mathrm{~h}$ and plated in triplicate at single cell density for clonogenic assay. Plates were incubated over 7-9 d, with media changed halfway through before colonies were stained with $0.1 \%$ Crystal Violet in $95 \%$ ethanol. Colonies were then manually counted. Cells that were infected for flow cytometry were selected and grown in drug-free medium for $24-48 \mathrm{~h}$ before being fixed in $70 \%$ ethanol at $-20^{\circ} \mathrm{C}$ for at least $24 \mathrm{~h}$. Cells were stained with phosphoSer10 Histone H3 (ab5176) and Goat-anti-rabbit AlexaFluor 488 (from Jackson Laboratories) for mitotic cells as well as propidium iodide (PI) for DNA content and run on a FACSCaliber flow cytometry machine. At least 10,000 cells were counted per experiment and three independent experiments were analyzed using Flowjo software.

\section{Cell growth experiments}

For HAP1 clonogenic assays, cells were seeded in 24-well dishes and transfected with LentiCRISPR v2 plasmids using XtremeGene 9 according to manufacturer's instructions. After $48 \mathrm{~h}$ of puromycin selection, cells were collected and the control counted so cells could 
be plated at single cell density in triplicate and assessed as described above. All other cell lines were infected with LentiCRISPRV2 containing guides targeting specific genes. After selection, cells were then grown in drug-free medium for another $24 \mathrm{~h}$ before cells were collected, counted, and re-plated at equal density across each cell line in triplicate in either 96 -well plates for nuclei counting or six-well dishes for clonogenic assay. For drug experiments, talozoparib, olaparib and bortezomib were from Selleck, deferoxamine (DFO), and thapsigargin were from Cedarlane Labs, and camptothecin (CPT), etoposide, hydroxyurea (HU), 5-fluorouracil (5-FU), SAHA, paclitaxel and anisomycin were from Sigma-Aldrich. To generate inhibition curves, cells were plated in 96-well dishes and drug was added the following day. Cells were incubated for a further 3-4 $d$ before nuclei were stained and counted. $I C_{50} \mathrm{~S}$ were calculated using GraphPad Prism v8. For serial dilution experiments, HAP1 cells were transfected with lentiCRISPR V2, selected with puromycin and the control counted to make a cell slurry where the control would reach $80-90 \%$ confluency in 7-9 d. Cells were serially diluted 1:2 and plated in 96-well dishes and allowed to grow for 7-9 $d$ before being stained with $0.1 \%$ crystal violet. $\mathrm{H} 4$ cells were infected with lentiCRISPR v2 constructs targeting specific genes, selected, counted and normalized before being serially diluted similar to HAP1 cells.

\section{Chromatin fractionation}

HAP1 WT and KO cells were transfected and selected as above before being moved to $25-\mathrm{cm}^{2}$ flasks with multiple wells of the same transfection pooled together. Transfections grew for another $4 \mathrm{~d}$ before cells were collected and fractionated with a protocol similar to Méndez and Stillman (2000). Briefly, 3-6 × $10^{6}$ cells were resuspended in Buffer A1 (10 mM Hepes, pH 7.9, 10 mM KCl, 1.5 mM MgCl $2,0.34$ M sucrose, $10 \%$ glycerol, $1 \mathrm{mM}$ DTT, and cOmplete protease inhibitors; Roche). An equal volume of Buffer A2 (Buffer A1 + 0.2\% Triton X-100) was added, and cells were incubated on ice for $\sim 7 \mathrm{~min}$. Cells were spun down at $1,300 \mathrm{~g}$ for 4 min at $4^{\circ} \mathrm{C}$ and the supernatant (S1) removed. The pellet (P1) was washed once with Buffer $A 1$ before being resuspended in Buffer $B$ ( 3 mM EDTA, 0.2 mM EGTA, and $1 \mathrm{mM}$ DTT + protease inhibitors). Cells were spun again at $1,700 \mathrm{~g}$ for 4 min at $4^{\circ} \mathrm{C}$ and the supernatant (S2) removed before the pellet (P2) was washed once with Buffer $\mathrm{B}$ and resuspended in $1 \times$ Laemmli (2\% SDS, 10\% glycerol, $60 \mathrm{mM}$ Tris, pH 6.8, $0.01 \mathrm{mg}$ bromophenol blue) and sonicated. For lysates, $\sim 4 \times 10^{6}$ cells from the same original cell slurry were lysed in $50 \mathrm{mM}$ Tris-HCl, pH7.5, $150 \mathrm{mM}$ $\mathrm{NaCl}, 3 \mathrm{mM}$ EDTA, 10\% glycerol, $1 \%$ Triton X-100, and protease inhibitors and sonicated. Both lysates and the $\mathrm{S} 1$ fraction were spun at $\sim 18,000 \mathrm{~g}$ at $4^{\circ} \mathrm{C}$ for $15 \mathrm{~min}$. The protein concentration of the lysate was determined using BCA (Thermo Fisher Scientific) and $20 \mu \mathrm{g}$ of each lysate, or the equivalent volume of S1 (soluble) and P2 (chromatin) fractions was used for Western blot.

\section{Western blots}

Except for those cells undergoing chromatin fractionation, all other samples for Western blot were lysed in Lysis buffer $(50 \mathrm{mM} \mathrm{Tris-HCl}$, $\mathrm{pH}$ 7.5, $150 \mathrm{mM} \mathrm{NaCl}, 10 \%$ glycerol, 1\% Triton X-100, and protease inhibitors), sonicated, and debris spun down as above. Samples were normalized by protein concentration using BCA, run on SDS-PAGE gels of appropriate acrylamide concentration and transferred to
PVDF membrane (Immobilon-FL; Millipore). After probing with primary and secondary antibodies, blots were then subjected to ECL (Clarity or Clarity Max Western ECL substrate; Bio-Rad) and visualized using a Bio-Rad ChemiDoc MP Imager in the appropriate channel. For Western blot quantification, bands were quantified using the Bio-Rad ImageLab software.

\section{Statistics}

With the exception of the bubble graphs in Fig 1, graphs and inhibition curves were generated using GraphPad Prism v8. Bubble graphs were generated with Microsoft Excel. Data points represent the mean and SEM unless otherwise indicated. P-values were calculated using a two-tailed, unpaired, Welch's $t$ test unless otherwise indicated.

\section{Supplementary Information}

Supplementary Information is available at https://doi.org/10.26508/lsa. 202101083.

\section{Acknowledgements}

We would like to thank Nigel O'Neil and Akil Hazma for discussion and review of the manuscript, Catherine Ross and Kevin Brown for help with data analysis, and Dr Todd Waldman for providing STAG2-modified cell lines. Funding was provided by a Canadian Institutes for Health Research grant to P Hieter (FDN 148449) and Canadian Institute for Advanced Research Genetic Networks Catalyst Funding to P Hieter and I Moffat.

\section{Author Contributions}

ML Bailey: conceptualization, data curation, formal analysis, investigation, methodology, and writing-original draft, review, and editing.

D Tieu: investigation and writing-review and editing.

A Habsid: investigation and writing-review and editing.

AHY Tong: investigation and writing-review and editing.

K Chan: investigation, methodology, and writing-review and editing. J Moffat: conceptualization, funding acquisition, investigation, and writing-review and editing.

P Hieter: conceptualization, funding acquisition, and writingreview and editing.

\section{Conflict of Interest Statement}

The authors declare that they have no conflict of interest.

\section{References}

Aregger M, Chandrashekhar M, Tong AHY, Chan K, Moffat J (2019) Pooled lentiviral CRISPR-Cas9 screens for functional genomics in mammalian cells. Methods Mol Biol 1869: 169-188. doi:10.1007/978-1-4939-88051_15 
Aregger M, Lawson KA, Billmann M, Costanzo M, Tong AHY, Chan K, Rahman M, Brown KR, Ross C, Usaj M, et al (2020) Systematic mapping of genetic interactions for de novo fatty acid synthesis identifies C12orf49 as a regulator of lipid metabolism. Nat Metab 2: 499-513. doi:10.1038/ s42255-020-0211-z

Bailey ML, O'Neil NJ, Van Pel DM, Solomon DA, Waldman T, Hieter P (2014) Glioblastoma cells containing mutations in the cohesin component STAG2 are sensitive to PARP inhibition. Mol Cancer Ther 13: 724-732. doi:10.1158/1535-7163.MCT-13-0749

Balbás-Martínez C, Sagrera A, Carrillo-De-Santa-Pau E, Earl J, Márquez M, Vazquez M, Lapi E, Castro-Giner F, Beltran S, Bayés M, et al (2013) Recurrent inactivation of STAG2 in bladder cancer is not associated with aneuploidy. Nat Genet 45: 1464-1469. doi:10.1038/ng.2799

Behan FM, Iorio F, Picco G, Gonçalves E, Beaver CM, Migliardi G, Santos R, Rao Y, Sassi F, Pinnelli M, et al (2019) Prioritization of cancer therapeutic targets using CRISPR-Cas9 screens. Nature 568: 511-516. doi:10.1038/ S41586-019-1103-9

Benedetti L, Cereda M, Monteverde L, Desai N, Ciccarelli FD, Benedetti L, Cereda M, Monteverde L, Desai N, Ciccarelli FD (2017) Synthetic lethal interaction between the tumour suppressor STAG2 and its paralog STAG1. Oncotarget 8: 37619-37632. doi:10.18632/oncotarget.16838

Benedict B, van Schie JJM, Oostra AB, Balk JA, Wolthuis RMF, Riele HT, de Lange J (2020) WAPL-dependent repair of damaged DNA replication forks underlies oncogene-induced loss of sister chromatid cohesion. Dev Cell 52: 683-698.e7. doi:10.1016/j.devcel.2020.01.024

Bisht KK, Daniloski Z, Smith S (2013) SA1 binds directly to DNA through its unique AT-hook to promote sister chromatid cohesion at telomeres. I Cell Sci 126: 3493-3503. doi:10.1242/jcs.130872

Brown KR, Mair B, Soste M, Moffat J (2019) CRISPR screens are feasible in TP53 wild-type cells. Mol Syst Biol 15: e8679. doi:10.15252/msb.20188679

Casa V, Moronta Gines M, Gade Gusmao E, Slotman JA, Zirkel A, Josipovic N, Oole E, Van ljcken WFJ, Houtsmuller AB, Papantonis A, et al (2020) Redundant and specific roles of cohesin STAG subunits in chromatin looping and transcriptional control. Genome Res 30: 515-527. doi:10.1101/gr.253211.119

Chaudhuri AR, Callen E, Ding X, Gogola E, Duarte AA, Lee JE, Wong N, Lafarga V, Calvo JA, Panzarino NJ, et al (2016) Replication fork stability confers chemoresistance in BRCA-deficient cells. Nature 539: 382-387. doi:10.1038/nature19826

Cho YW, Hong T, Hong S, Guo H, Yu H, Kim D, Guszczynski T, Dressler GR, Copeland TD, Kalkum M, et al (2007) PTIP associates with MLL3- and MLL4-containing histone H3 lysine 4 methyltransferase complex. J Biol Chem 282: 20395-20406. doi:10.1074/jbc.M701574200

Countryman P, Fan Y, Gorthi A, Pan H, Strickland J, Kaur P, Wang X, Lin J, Lei X, White C, et al (2018) Cohesin SA2 is a sequence-independent DNAbinding protein that recognizes DNA replication and repair intermediates. J Biol Chem 293: 1054-1069. doi:10.1074/ jbc.M117.806406

Daniloski Z, Smith S (2017) Loss of tumor suppressor STAG2 promotes telomere recombination and extends the replicative lifespan of normal human cells. Cancer Res 77: 5530-5542. doi:10.1158/00085472.CAN-17-1260

D'Antonio M, Guerra RF, Cereda M, Marchesi S, Montani F, Nicassio F, Di Fiore PP, Ciccarelli FD (2013) Recessive cancer genes engage in negative genetic interactions with their functional paralogs. Cell Rep 5: 1519-1526. doi:10.1016/j.celrep.2013.11.033

Dede M, McLaughlin M, Kim E, Hart T (2020) Multiplex enCas12a screens detect functional buffering among paralogs otherwise masked in monogenic Cas9 knockout screens. Genome Biol 21: 262. doi:10.1186/s13059-02002173-2

Galeev R, Baudet A, Kumar P, Rundberg Nilsson A, Nilsson B, Soneji S, Törngren T, Borg A, Kvist A, Larsson J (2016) Genome-wide RNAi screen identifies cohesin genes as modifiers of renewal and differentiation in human HSCs. Cell Rep 14: 2988-3000. doi:10.1016/j.celrep.2016. 02.082

Gonatopoulos-Pournatzis T, Aregger M, Brown KR, Farhangmehr S, Braunschweig U, Ward HN, Ha KCH, Weiss A, Billmann M, Durbic T, et al (2020) Genetic interaction mapping and exon-resolution functional genomics with a hybrid Cas9-Cas12a platform. Nat Biotechnol 38: 638-648. doi:10.1038/s41587-020-0437-z

Haarhuis JH, Elbatsh AM, Van Den Broek B, Camps D, Erkan H, Jalink K, Medema RH, Rowland BD (2013) WAPL-mediated removal of cohesin protects against segregation errors and aneuploidy. Curr Biol 23: 2071-2077. doi:10.1016/j.cub.2013.09.003

Haarhuis JHI, van der Weide RH, Blomen VA, Yáñez-Cuna JO, Amendola M, van Ruiten MS, Krijger PHL, Teunissen H, Medema RH, van Steensel B, et al (2017) The cohesin release factor WAPL restricts chromatin loop extension. Cell 169: 693-707.e14. doi:10.1016/j.cell.2017.04.013

Hart T, Brown KR, Sircoulomb F, Rottapel R, Moffat I (2014) Measuring error rates in genomic perturbation screens: Gold standards for human functional genomics. Mol Syst Biol 10: 733. doi:10.15252/msb.20145216

Hart T, Chandrashekhar M, Aregger M, Steinhart Z, Brown KR, MacLeod G, Mis M, Zimmermann M, Fradet-Turcotte A, Sun S, et al (2015) Highresolution CRISPR screens reveal fitness genes and genotype-specific cancer liabilities. Cell 163: 1515-1526. doi:10.1016/j.cell.2015.11.015

Hart T, Tong AHY, Chan K, Van Leeuwen J, Seetharaman A, Aregger M, Chandrashekhar M, Hustedt N, Seth S, Noonan A, et al (2017) Evaluation and design of genome-wide CRISPR/SpCas9 knockout screens. G3 (Bethesda) 7: 2719-2727. doi:10.1534/g3.117.041277

Heidinger-Pauli JM, Mert O, Davenport C, Guacci V, Koshland D (2010) Systematic reduction of cohesin differentially affects chromosome segregation, condensation, and DNA repair. Curr Biol 20: 957-963. doi:10.1016/j.cub.2010.04.018

Hill VK, Kim JS, Waldman T (2016) Cohesin mutations in human cancer. Biochim Biophys Acta, 1866: 1-11. doi:10.1016/j.bbcan.2016.05.002

Iorio F, Knijnenburg TA, Vis DJ, Bignell GR, Menden MP, Schubert M, Aben N, Gonçalves E, Barthorpe S, Lightfoot H, et al (2016) A landscape of pharmacogenomic interactions in cancer. Cell 166: 740-754. doi:10.1016/j.cell.2016.06.017

Katsarou A, Pantopoulos K (2020) Basics and principles of cellular and systemic iron homeostasis. Mol Aspects Med 75: 100866. doi:10.1016/ j.mam.2020.100866

Kawauchi S, Calof AL, Santos R, Lopez-Burks ME, Young CM, Hoang MP, Chua A, Lao T, Lechner MS, Daniel JA, et al (2009) Multiple organ system defects and transcriptional dysregulation in the $\mathrm{Nipbl}(+/-)$ mouse, a model of Cornelia de Lange Syndrome. PLoS Genet 5: e1000650. doi:10.1371/ journal.pgen.1000650

Kim E, Dede M, Lenoir WF, Wang G, Srinivasan S, Colic M, Hart T (2019) A network of human functional gene interactions from knockout fitness screens in cancer cells. Life Sci Alliance 2: e201800278. doi:10.26508/ Isa.201800278

Kojic A, Cuadrado A, De Koninck M, Giménez-Llorente D, Rodríguez-Corsino M, Gómez-López G, Le Dily F, Marti-Renom MA, Losada A (2018) Distinct roles of cohesin-SA1 and cohesin-SA2 in 3D chromosome organization. Nat Struct Mol Biol 25: 496-504. doi:10.1038/s41594-0180070-4

Kong X, Ball AR, Pham HX, Zeng W, Chen HY, Schmiesing JA, Kim JS, Berns M, Yokomori K (2014) Distinct functions of human cohesin-SA1 and cohesin-SA2 in double-strand break repair. Mol Cell Biol 34: 685-698. doi:10.1128/MCB.01503-13

Kontoghiorghe CN, Kontoghiorghes GJ (2016) Efficacy and safety of ironchelation therapy with deferoxamine, deferiprone, and deferasirox for the treatment of iron-loaded patients with non-transfusiondependent thalassemia syndromes. Drug Des Devel Ther 10: 465-481. doi:10.2147/DDDT.S79458 
Kühn LC (2015) Iron regulatory proteins and their role in controlling iron metabolism. Metallomics 7: 232-243. doi:10.1039/c4mt00164h

Lawrence MS, Stojanov P, Mermel CH, Robinson JT, Garraway LA, Golub TR, Meyerson M, Gabriel SB, Lander ES, Getz G (2014) Discovery and saturation analysis of cancer genes across 21 tumour types. Nature 505: 495-501. doi:10.1038/nature12912

Lechner MS, Levitan I, Dressler GR (2000) PTIP, a novel BRCT domain-containing protein interacts with Pax2 and is associated with active chromatin. Nucleic Acids Res 28: 2741-2751. doi:10.1093/nar/28.14.2741

Liu J, Zhang Z, Bando M, Itoh T, Deardorff MA, Clark D, Kaur M, Tandy S, Kondoh T, Rappaport E, et al (2009) Transcriptional dysregulation in NIPBL and cohesin mutant human cells. PLOS Biol 7: e1000119. doi:10.1371/ journal.pbio.1000119

Liu Y, Xu H, Van Der Jeught K, Li Y, Liu S, Zhang L, Fang Y, Zhang X, Radovich M, Schneider BP, et al (2018) Somatic mutation of the cohesin complex subunit confers therapeutic vulnerabilities in cancer. J Clin Invest 128: 2951-2965. doi:10.1172/JCI98727

Lord CJ, Quinn N, Ryan CJ (2020) Integrative analysis of large-scale loss-offunction screens identifies robust cancer-associated genetic interactions. Elife 9: 1-37. doi:10.7554/eLife.58925

Losada A (2014) Cohesin in cancer: Chromosome segregation and beyond. Nat Rev Cancer 14: 389-393. doi:10.1038/nrc3743

Mair B, Moffat J, Boone C, Andrews BJ (2019) Genetic interaction networks in cancer cells. Curr Opin Genet Dev 54: 64-72. doi:10.1016/ j.gde.2019.03.002

McLellan JL, O'Neil NJ, Barrett I, Ferree E, van Pel DM, Ushey K, Sipahimalani P, Bryan J, Rose AM, Hieter P (2012) Synthetic lethality of cohesins with PARPs and replication fork mediators. PLoS Genet 8: e1002574. doi:10.1371/journal.pgen.1002574

Méndez J, Stillman B (2000) Chromatin association of human origin recognition complex, $\mathrm{Cdc} 6$, and minichromosome maintenance proteins during the cell cycle: Assembly of prereplication complexes in late mitosis. Mol Cell Biol 20: 8602-8612. doi:10.1128/MCB.20.22.86028612.2000

Meyron-Holtz EG, Ghosh MC, Iwai K, LaVaute T, Brazzolotto X, Berger UV, Land W, Ollivierre-Wilson H, Grinberg A, Love P, et al (2004) Genetic ablations of iron regulatory proteins 1 and 2 reveal why iron regulatory protein 2 dominates iron homeostasis. EMBO J 23: 386-395. doi:10.1038/sj.emboj.7600041

Mondal G, Stevers M, Goode B, Ashworth A, Solomon DA (2019) A requirement for STAG2 in replication fork progression creates a targetable synthetic lethality in cohesin-mutant cancers. Nat Commun 10: 1686. doi:10.1038/s41467-019-09659-z

Mullenders J, Aranda-Orgilles B, Lhoumaud P, Keller M, Pae J, Wang K, Kayembe C, Rocha PP, Raviram R, Gong Y, et al (2015) Cohesin loss alters adult hematopoietic stem cell homeostasis, leading to myeloproliferative neoplasms. J Exp Med 212: 1833-1850. doi:10.1084/ jem.20151323

Muller FL, Colla S, Aquilanti E, Manzo VE, Genovese G, Lee J, Eisenson D, Narurkar R, Deng P, Nezi L, et al (2012) Passenger deletions generate therapeutic vulnerabilities in cancer. Nature 488: 337-342. doi:10.1038/nature11331

Newkirk DA, Chen YY, Chien R, Zeng W, Biesinger J, Flowers E, Kawauchi S, Santos R, Calof AL, Lander AD, et al (2017) The effect of Nipped-B-like (Nipbl) haploinsufficiency on genome-wide cohesin binding and target gene expression: Modeling Cornelia de Lange syndrome. Clin Epigenetics 9: 89. doi:10.1186/s13148-017-0391-x

O'Neil NJ, Bailey ML, Hieter P (2017) Synthetic lethality and cancer. Nat Rev Genet 18: 613-623. doi:10.1038/nrg.2017.47

Pan J, Meyers RM, Michel BC, Mashtalir N, Sizemore AE, Wells JN, Cassel SH, Vazquez F, Weir BA, Hahn WC, et al (2018) Interrogation of mammalian protein complex structure, function, and membership using genome- scale fitness screens. Cell Syst 6: 555-568.e7. doi:10.1016/ j.cels.2018.04.011

Patel SR, Kim D, Levitan I, Dressler GR (2007) The BRCT-domain containing protein PTIP links PAX2 to a histone H3, lysine 4 methyltransferase complex. Dev Cell 13: 580-592. doi:10.1016/ j.devcel.2007.09.004

Pherson M, Misulovin Z, Gause M, Dorsett D (2019) Cohesin occupancy and composition at enhancers and promoters are linked to DNA replication origin proximity in Drosophila. Genome Res 29: 602-612. doi:10.1101/gr.243832.118

Remeseiro S, Cuadrado A, Kawauchi S, Calof AL, Lander AD, Losada A (2013) Reduction of Nipbl impairs cohesin loading locally and affects transcription but not cohesion-dependent functions in a mouse model of Cornelia de Lange Syndrome. Biochim Biophys Acta 1832: 2097-2102. doi:10.1016/j.bbadis.2013.07.020

Rollins RA, Korom M, Aulner N, Martens A, Dorsett D (2004) Drosophila nipped-B protein supports sister chromatid cohesion and opposes the stromalin/ $\mathrm{scc} 3$ cohesion factor to facilitate long-range activation of the cut gene. Mol Cell Biol 24: 3100-3111. doi:10.1128/mcb.24.8.31003111.2004

Rubio ED, Reiss DJ, Welcsh PL, Disteche CM, Filippova GN, Baliga NS, Aebersold R, Ranish JA, Krumm A (2008) CTCF physically links cohesin to chromatin. Proc Natl Acad Sci U S A 105: 8309-8314. doi:10.1073/ pnas.0801273105

Ryan CJ, Bajrami I, Lord CJ (2018) Synthetic lethality and cancer: Penetrance as the major barrier. Trends Cancer 4: 671-683. doi:10.1016/ j.trecan.2018.08.003

Sarogni P, Pallotta MM, Musio A (2020) Cornelia de Lange syndrome: From molecular diagnosis to therapeutic approach. J Med Genet 57: 289-295. doi:10.1136/jmedgenet-2019-106277

Schwab KR, Patel SR, Dressler GR (2011) Role of PTIP in class switch recombination and long-range chromatin interactions at the immunoglobulin heavy chain locus. Mol Cell Biol 31: 1503-1511. doi:10.1128/MCB.00990-10

Shen JP, Ideker T (2018) Synthetic lethal networks for precision oncology: Promises and pitfalls. J Mol Biol 430: 2900-2912. doi:10.1016/ j.jmb.2018.06.026

Shen JP, Zhao D, Sasik R, Luebeck J, Birmingham A, Bojorquez-Gomez A, Licon K, Klepper K, Pekin D, Beckett AN, et al (2017) Combinatorial CRISPRCas9 screens for de novo mapping of genetic interactions. Nat Methods 14: 573-576. doi:10.1038/nmeth.4225

Smith SR, Ghosh MC, Ollivierre-Wilson H, Hang Tong W, Rouault TA (2006) Complete loss of iron regulatory proteins 1 and 2 prevents viability of murine zygotes beyond the blastocyst stage of embryonic development. Blood Cells Mol Dis 36: 283-287. doi:10.1016/ j.bcmd.2005.12.006

Solomon DA, Kim T, Diaz-Martinez LA, Fair J, Elkahloun AG, Harris BT, Toretsky JA, Rosenberg SA, Shukla N, Ladanyi M, et al (2011) Mutational inactivation of STAG2 causes aneuploidy in human cancer. Science 333: 1039-1043. doi:10.1126/science.1203619

Starnes LM, Su D, Pikkupeura LM, Weinert BT, Santos MA, Mund A, Soria R, Cho YW, Pozdnyakova I, Kubec Højfeldt M, et al (2016) A PTIP-PA1 subcomplex promotes transcription for IgH class switching independently from the associated MLL3/MLL4 methyltransferase complex. Genes Dev 30: 149-163. doi:10.1101/gad.268797.115

Torti SV, Torti FM (2013) Iron and cancer: More ore to be mined. Nat Rev Cancer 13: 342-355. doi:10.1038/nrc3495

Tsherniak A, Vazquez F, Montgomery PG, Weir BA, Kryukov G, Cowley GS, Gill S, Harrington WF, Pantel S, Krill-Burger JM, et al (2017) Defining a cancer dependency Map. Cell 170: 564-576.e16. doi:10.1016/j.cell.2017.06.010

Uhlmann F (2016) SMC complexes: From DNA to chromosomes. Nat Rev Mol Cell Biol 17: 399-412. doi:10.1038/nrm.2016.30 
van der Lelij P, Lieb S, Jude J, Wutz G, Santos CP, Falkenberg K, Schlattl A, Ban J, Schwentner R, Hoffmann T, et al (2017) Synthetic lethality between the cohesin subunits STAG1 and STAG2 in diverse cancer contexts. Elife 6: e26980. doi:10.7554/eLife.26980

van der Lelij P, Newman JA, Lieb S, Jude J, Katis V, Hoffmann T, Hinterndorfer M, Bader G, Kraut N, Pearson MA, et al (2020) STAG1 vulnerabilities for exploiting cohesin synthetic lethality in STAG2-deficient cancers. Life Sci Alliance 3: e202000725. doi:10.26508/lsa.202000725

Viny AD, Bowman RL, Liu Y, Lavallée VP, Eisman SE, Xiao W, Durham BH, Navitski A, Park J, Braunstein S, et al (2019) Cohesin members Stag1 and Stag2 display distinct roles in chromatin accessibility and topological control of HSC self-renewal and differentiation. Cell Stem Cell 25: 682-696.e8. doi:10.1016/j.stem.2019.08.003

Waldman T (2020) Emerging themes in cohesin cancer biology. Nat Rev Cancer 20: 504-515. doi:10.1038/s41568-020-0270-1

Wang C, Wang G, Feng X, Shepherd P, Zhang J, Tang M, Chen Z, Srivastava M, McLaughlin ME, Navone NM, et al (2019) Genome-wide CRISPR screens reveal synthetic lethality of RNASEH2 deficiency and ATR inhibition. Oncogene 38: 2451-2463. doi:10.1038/s41388-018-0606-4

Wang W, Di X, D'Agostino RB, Torti SV, Torti FM (2007) Excess capacity of the iron regulatory protein system. J Biol Chem 282: 24650-24659. doi:10.1074/jbc.M703167200

Wong AS, Choi GC, Cui CH, Pregernig G, Milani P, Adam M, Perli SD, Kazer SW, Gaillard A, Hermann M, et al (2016) Multiplexed barcoded CRISPR-Cas9 screening enabled by CombiGEM. Proc Natl Acad Sci U S A 113: 2544-2549. doi:10.1073/pnas.1517883113
Wu J, Prindle MJ, Dressler GR, Yu X (2009) PTIP regulates 53BP1 and SMC1 at the DNA damage sites. J Biol Chem 284: 18078-18084. doi:10.1074/ jbc.M109.002527

Xiao T, Wallace J, Felsenfeld G (2011) Specific sites in the C terminus of CTCF interact with the SA2 subunit of the cohesin complex and are required for cohesin-dependent insulation activity. Mol Cell Biol 31: 2174-2183. doi:10.1128/MCB.05093-11

Yaguchi K, Yamamoto T, Matsui R, Tsukada Y, Shibanuma A, Kamimura K, Koda $T$, Uehara R (2018) Uncoordinated centrosome cycle underlies the instability of non-diploid somatic cells in mammals. J Cell Biol 217: 2463-2483. doi:10.1083/jcb.201701151

Yang W, Soares J, Greninger P, Edelman EJ, Lightfoot H, Forbes S, Bindal N, Beare D, Smith JA, Thompson IR, et al (2013) Genomics of drug sensitivity in cancer (GDSC): A resource for therapeutic biomarker discovery in cancer cells. Nucleic Acids Res 41: D955-D961. doi:10.1093/ nar/gks1111

Zimmermann M, Murina O, Reijns MAM, Agathanggelou A, Challis R, Tarnauskaite Ž, Muir M, Fluteau A, Aregger M, McEwan A, et al (2018) CRISPR screens identify genomic ribonucleotides as a source of PARP-trapping lesions. Nature 559: 285-289. doi:10.1038/s41586-018-0291-z

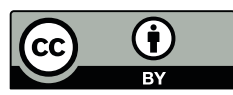

License: This article is available under a Creative Commons License (Attribution 4.0 International, as described at https://creativecommons.org/ licenses/by/4.0/). 